\title{
COMbIEN DE NÉERLANDAIS ? \\ Histoire linguistiQue ET HISTOIRE DE LA LinguistiQue DANS LES ÎLES VIERGES DANOISES
}

\author{
Philipp Krämer
}

Freie Universität Berlin

\section{Résumé}

La langue créole à base néerlandaise des Îles Vierges danoises a été documentée pendant des siècles non par des Néerlandais, mais par des missionnaires allemands et des fonctionnaires coloniaux danois. Cet article a pour objectif de retracer la place du néerlandais dans cet univers colonial complexe. Nous examinerons des sources historiques des $\mathrm{XVIII}^{\mathrm{e}}$ et $\mathrm{XIX}^{\mathrm{e}}$ siècles pour déterminer le rôle sociolinguistique de la langue néerlandaise et du créole avant de dépister des indices sur les connaissances (méta-)linguistiques du néerlandais des créolistes (C.G.A. Oldendorp, J. M. Magens et E. Pontoppidan). En outre, nous offrirons quelques réflexions sur les fondements discursifs et épistémologiques et la place que les auteurs accordent à la langue de base du créole local. Nous montrerons que les complexités linguistique et métalinguistique de cet archipel sont bien différentes de la plupart des autres colonies créolophones.

\section{Mots-clés}

Colonialisme, mission chrétienne, langues créoles, cariole ( « Negerhollands »), néerlandais, universalisme, racialisme, Îles Vierges danoises

\section{Abstract}

For centuries, the Dutch-based Creole language of the Danish Virgin Islands was documented not by the Dutch but mainly by German missionaries and Danish colonialists. This article sheds light on the role of the Dutch language in this complex colonial universe. Historical sources from the 18th and 19th century will show which sociolinguistic role Dutch played in the society of the islands and which (meta-)linguistic knowledge of Dutch the authors of these sources (C.G.A. Oldendorp, J. M. Magens, and E. Pontoppidan) had. Some reflections on the discursive and epistemological foundations of the sources and the significance they attribute to the Dutch language will conclude the article in order to show that the linguistic complexities of this archipelago are different from most other Creole-speaking areas.

\section{Keywords}

Colonialism, Christian mission, Creole languages, cariole (« Negerhollands »), Dutch, universalism, racialism, Danish Virgin Islands 
Dans l'histoire coloniale du monde, les Îles Vierges représentent un cas particulier dans la mesure où une partie de ce petit archipel a connu une longue période sous le contrôle du Danemark ${ }^{1}$. Depuis la fin du XVII ${ }^{\mathrm{e}}$ s., les deux îles de Saint-Thomas et de Saint-John (Saint-Jean) étaient sous administration permanente danoise ; l'île de Sainte-Croix les rejoignit quelques décennies plus tard. En dépit d'un changement de statut administratif - avant de devenir colonies du Royaume de Danemark, les îles étaient des possessions de la Compagnie danoise des Indes occidentales - la période danoise dura jusqu'à la vente définitive du territoire aux États-Unis en 1917. Si le Danemark ne comptait pas parmi les grandes puissances mondiales malgré son rôle régional dans le nord de l'Europe, ses activités coloniales ne furent ni éphémères, ni tardives. Par cette présence durable sur la scène coloniale, le Danemark se différencie de l'histoire coloniale allemande, italienne ou belge.

Outre cette singularité historique, il faut noter une particularité linguistique qui fait des Îles Vierges danoises un lieu d'étude privilégié de la recherche sur les langues créoles. À cause des contacts soutenus avec les Pays-Bas et leurs colonies insulaires dans la région caribéenne, c'est dans ces îles qu'a pu se former l'une des langues créoles à base néerlandaise ${ }^{2}$, groupe qui ne compte que deux autres variétés relativement stables, le Skepi Dutch et le Berbice Dutch, autrefois parlés au Guyana3. Si toutes ces langues ont désormais disparu (ou presque : une dernière locutrice du Berbice Dutch serait encore en vie), la langue créole néerlandaise des Îles Vierges est particulièrement bien documentée.

Cette langue est connue aujourd'hui encore sous le nom de Negerhollands, terme courant depuis le $\mathrm{XIX}^{\mathrm{e}} \mathrm{s}$. Si ce terme est toujours utilisé par les linguistes et qu'aucun autre n'a réussi à s'imposer, la communauté scientifique est consciente des problèmes posés par sa connotation raciste et son imprécision historique : dans la période de formation de cette langue, les dialectes prédominants étaient ceux de la Flandre occidentale et de la Zélande tandis que les dialectes hollandais jouaient un rôle assez limité (Van Rossem 2000). Nous opterons ici pour une solution provisoire en proposant le terme cariole, néologisme formé par francisation du mot allemand Cariolisch ou carriols que l'on trouve notamment dans des lettres et textes liturgiques émanant de la mission protestante au milieu du XVIII ${ }^{\mathrm{e}} \mathrm{s}{ }^{4}$. Ce terme est antérieur à celui de Negerhollands et il présente l'avantage de signifier créole,

1 Je tiens à exprimer mes remerciements à Truus De Wilde, Matthias Hüning, Jan Noordegraaf, Pascale Rabault-Feuerhahn, Peter Stein, Cefas van Rossem et Magdalena von Sicard pour leurs conseils et corrections.

2 Voir Van Rossem (2000, p. 46-47) pour les différentes hypothèses sur la genèse de cette langue.

3 Nous laisserons de côté, dans le cadre de ce travail, la discussion autour de l'Afrikaans (ou de son précurseur colonial) en tant que langue créole, sémi-créole ou autrement restructurée, voir à ce propos Markey (1982); Holm (2000, p. 51, 81); Roberge (2002).

4 Voir les différents documents cités et édités dans Van Rossem / Van der Voort (1996, p. 49-50, $71,93,286,307)$. Pour les mentions du terme allemand carriol(se) dans les premières sources, voir Stein (2013, p. 55-56). 
sans aucune connotation secondaire, tout en étant propre à cette seule langue car la forme cariole n'est utilisée que dans des documents portant sur la langue créole à base néerlandaise des Îles Vierges.

L'objet de cet article est de retracer le rôle de la langue néerlandaise dans l'histoire linguistique des Îles Vierges danoises et notamment dans l'histoire de la recherche philologique ou linguistique « locale », c'est-à-dire dans les descriptions grammaticales du cariole établies par des missionnaires européens installés dans l'archipel au XVIII ${ }^{\mathrm{e}}$ s. ainsi que dans les travaux de leurs successeurs.

Nous nous appuierons pour ce faire sur deux grammaires du cariole parues vers la fin $\mathrm{du}_{\mathrm{XVIII}}^{\mathrm{e}} \mathrm{s}$. La première est contenue dans un rapport très détaillé que l'Allemand Christian Georg Andreas Oldendorp (1721-1787), membre de la communauté des Frères moraves (Herrnhuter Brüdergemeine), écrivit sur leurs activités dans les Îles Vierges. Contenant aussi des informations sur l'histoire de la mission et des observations ethnologiques, le livre parut en $1777^{6}$. L'autre grammaire avait été publiée sept ans plus tôt par le Danois Jochum Melchior Magens, philologue et fonctionnaire local né à Saint-Thomas? ${ }^{7}$. À ces deux sources très complètes s'ajoute un petit article du médecin danois Erik Pontoppidan (1847$1919)^{8}$ dont parurent une version allemande (1881) et une version danoise (1887), qui ne sont pas parfaitement identiques. ${ }^{9}$

Ces trois textes fournissent des informations métalinguistiques qui permettent d'interroger la place du néerlandais dans la société coloniale des îles aussi bien que dans l'histoire de la recherche sur le cariole. Ils révèlent une situation complexe, dans laquelle la langue néerlandaise exerça des effets sous-jacents mais durables aussi bien sur les habitants des îles que sur les travaux des missionnaires et chercheurs, sans jamais entrer pour autant dans une position de prédominance ouverte.

\section{UN UNIVERS 《 PAN-GERMANIQUE »}

La continuité administrative du règne colonial danois dans les Îles Vierges ne doit pas faire oublier que la population des îles était très hétérogène. Cela se traduisait

5 Pour plus de détails biographiques, voir Gilbert (1994), Stein (1998), Oldendorp (2010 [1787]). Pour le rôle des Frères moraves dans l'histoire coloniale, notamment leurs rapports avec le colonialisme et l'esclavagisme néerlandais, voir Lampe (2001, p. 36-50), Rupp-Eisenreich (1985).

6 Dans la première publication en 1777, l'éditeur avait omis de grandes parties, notamment dans la grammaire du créole (Stein 2010, p. 215-216). Le manuscrit complet a été réédité et publié en 2000. Toutes les citations dans cet article sont tirées de cette édition avec la pagination originale du manuscrit entre parenthèses. Stein (2002) donne un aperçu de la composition de l'ouvrage et de sa réédition.

7 Voir les informations biographiques dans Van Rossem / Van der Voort (1996, p. 29-30) ainsi que la note dans Stein (1998).

8 Voir les informations biographiques plus détaillées dans Stein (2014, p. 109-110).

9 Voir les rééditions de ces deux textes dans Krämer (éd., 2014). 
par un très fort multilinguisme de la société tant parmi les colonisés que parmi les colonisateurs. Cette situation n'était certes pas exceptionnelle, surtout aux débuts de l'expansion européenne. Dans les Îles Vierges et les autres colonies, c'est notamment le système esclavagiste qui entraînait la présence d'une multitude d'autres langues, souvent africaines, auxquelles s'ajoutèrent plus tard les langues des travailleurs asiatiques engagés après l'abolition de l'esclavage. Mais dans la plupart des autres colonies, le multilinguisme s'était amenuisé à mesure qu'une puissance européenne s'était établie au détriment des colonisateurs d'autres nationalités européennes.

Oldendorp donne des informations détaillées sur la fonction des diverses langues européennes dans les îles ainsi que sur leur position vis-à-vis du créole ${ }^{10}$ :

Dans ces îles, on parle anglais, allemand, danois, hollandais, français, espagnol et créole. Mais l'anglais et le haut-allemand sont les langues dominantes. Avec elles, on arrive à se débrouiller partout. On a besoin du créole parmi les nègres. Les créoles et les habitants blancs du pays le connaissent aussi. Cependant, tous n'aiment pas le parler avec les Blancs parce qu'il est en même temps la langue des Nègres. Le danois, le hollandais et le français sont parlés par ces nations entre elles. L'espagnol se parle avant tout avec les Espagnols qui font du commerce ici. (Oldendorp 2000a [1777], p. 357 [373]) ${ }^{11}$

Il est intéressant d'observer que, selon Oldendorp, les langues prédominantes sont l'anglais et l'allemand, donc ni la langue de la puissance coloniale, ni celle dont la langue créole locale est dérivée. Le danois et le néerlandais ainsi que le français restent cantonnés à la communication au sein des communautés européennes de leurs locuteurs respectifs. Cette analyse est confirmée par Magens qui met lui aussi l'accent sur la diversité linguistique dans les îles :

En ce qui concerne la langue créole qui est utilisée sur toutes les îles antillaises, il faut observer que chaque nation a sa langue à part et que par conséquent elles ne peuvent pas se comprendre.

La langue créole utilisée sur les îles danoises est dérivée du hollandais parce que les premiers habitants venaient pour la plupart des provinces hollandaises ${ }^{12} \mathrm{en}$ Europe. (Magens 1770, p. 7) ${ }^{13}$

10 Voir aussi l'analyse des informations sociolinguistiques du travail d'Oldendorp dans Stein (2010, p. 229-231).

11 « Auf diesen Inseln wird englisch, deutsch, dänisch, holländisch, französisch, spanisch und criolisch geredet. Englisch und Hochdeutsch sind aber die dominanten Sprachen. Mit diesen kann man allenthalben durchkommen. Criolisch braucht man unter den Negern. Es könnens auch die Criolen und meisten weißen Einwohner des Landes. Sie reden es aber nicht alle gern mit Blanken, weil es zugleich die Negersprache ist. Dänisch, holländisch und französisch reden diese Nationen untereinander. Spanisch wird hauptsächlich mit denen dahin handelnden Spaniern gesprochen ». Toutes les traductions sont les miennes.

12 Magens parle ici de la République des sept Provinces-Unies des Pays-Bas et non pas de la seule région de la Hollande (provinces actuelles de Hollande-Septentrionale et Hollande-Méridionale), cf. plus haut les remarques sur les influences dialectales des premiers colonisateurs originaires de la Zélande et de la Flandre.

13 « Hvad sig angaaer det Creolske Sprog, som bruges paa alle de Vestindiske Eilande, da maa der observeres, at enhver Nation have sit a parte Sprog, saa at de følgelig ey kan forstaae 
Magens note aussi que les Néerlandais étaient le groupe démographiquement majoritaire au début de l'ère coloniale, ce qui explique selon lui l'émergence d'une langue créole à base néerlandaise plutôt que danoise ${ }^{14}$. Cependant, la prédominance néerlandaise n'était que relative car le nombre d'esclaves avait dépassé la population européenne dès la fin du XVII ${ }^{\mathrm{e}}$ s. (Stein 1996, p. 5 ; Sabino 2012, p. 63). Bien que le français et l'espagnol soient mentionnés, ce sont différentes branches de langues germaniques qui décidèrent du destin linguistique des trois îles. L'allemand (dans le contexte de la mission) et, à terme, l'anglais devinrent les langues socialement dominantes dans cette colonie danoise d'expression cariole - il ne faut pas oublier que l'autre moitié de l'archipel des Îles Vierges était une colonie britannique (aujourd'hui territoire d'outre-mer du Royaume-Uni), ni que la partie danoise est désormais sous administration américaine.

Dans cette situation complexe où le néerlandais restait assez marginal et où le danois était quasiment absent de la communication interethnique, le cariole s'est émancipé de sa langue de base en assumant une fonction très nette de langue véhiculaire entre les esclaves et les Européens dans tous les domaines, y compris la mission, et comme langue courante au sein de la communauté des esclaves. Cependant, un siècle après la parution des travaux de Magens et d'Oldendorp, Erik Pontoppidan émit des avis très catégoriques à propos de la situation sociolinguistique vers la fin du $\mathrm{XIX}^{\mathrm{e}} \mathrm{s}$. :

Aujourd'hui la connaissance du créole a quasiment disparu complètement à Ste. Croix; dans la ville de St. Thomas, on ne trouve qu'ici ou là quelques vieilles femmes nègres qui savent encore le parler, et sporadiquement des créoles blancs de la génération plus âgée qui s'en souviennent de leur enfance. À l'extérieur, parmi les nègres ruraux, il s'est mieux maintenu, et aussi sur l'île plus lointaine et préservée de St. Jean, on peut encore entendre des vieillards se servir du « Kreol-Tael » comme langue courante, peut-être même avec plus de passion et de pratique que l'anglais. Mais pour la génération plus jeune, même là c'est une langue morte, et le temps n'est pas loin où elle sera partout complètement oubliée et où elle ne survivra que dans quelques noms locaux et peut-être dans quelques vieux proverbes ou tournures. (Pontoppidan 2014 [1887], p. 90-91 [297])15

hverandre. / Det brugelige Creolske Sprog paa de Danske Eilande er deriveret af det Hollandske, af Aarsage, at de første Indvaanere vare mestendeels fra de Hollandske Provincer i Europa. »

14 Voir Sabino (2012, p. 59-71), Van Rossem (2013) pour une analyse plus détaillée et nuancée de l'histoire sociale des îles et le développement du cariole.

15 « For Øjeblikket er Kendskaben til Kreolsk saa godt som fuldstændigt forsvunden paa St. Croix; i Byen St. Thomas finder man kun hist og her nogle gamle Negerkvinder, som endnu kunne tale det, og af de hvide Kreoler enkelte af den ældre Slægt, der erindre det fra deres Barndom. Ude blandt Landnegrene har det dog holdt sig bedre, og paa den mere afsides og uberørte Ø St. Jan kan man endnu høre gamle Folk betjæne sig af "Kreol-Tael" som Omgangssprog, maaske endog med større Forkærlighed og Øvelse end Engelsk. Men for den yngre Generation er det selv dér et dødt Sprog, og den Tid er ikke fjærn, da det overalt vil være fuldstændig forglemt og kun leve i nogle Stednavne og maaske i et Par gamle Ordsprog og Talemaader. » 
Ces trois sources n'offrent pas seulement des informations sur la place du néerlandais dans la société des Antilles danoises, mais aussi sur la vitalité et le déclin du cariole. En comparant les témoignages de Pontoppidan et d'Oldendorp et en tenant compte de la date de décès de la dernière locutrice connue en 1987 , ces textes historiques nous renseignent aussi sur le rythme et les conditions de disparition d'une langue. Il est remarquable que le cariole ait perduré encore exactement cent ans après l'annonce de sa disparition imminente par Pontoppidan.

\section{CES « CRÉOLISTES » QUI N’ÉTAIENT PAS « NÉERLANDISTES »}

Pour travailler, comme Oldendorp, dans la mission chrétienne, il n'était pas indispensable, après la période initiale, de connaître le néerlandais puisque les textes liturgiques étaient désormais disponibles en traduction créole. De même, Magens n'avait sans doute besoin que du danois dans le contexte de l'administration coloniale et, dans une moindre mesure, du cariole et peut-être de l'anglais pour les échanges quotidiens. Il recommande pourtant de se familiariser avec le néerlandais si l'on veut rapidement apprendre le cariole :

Puisque cette langue créole est dérivée du hollandais, c'est une nécessité que celui qui veut rapidement apprendre cette première comprenne cette dernière ; c'est la raison pour laquelle ceux qui arrivent ici en provenance de contrées hollandaises peuvent apprendre à manier et à comprendre les nègres plus tôt que ceux qui viennent d'ailleurs. (Magens 1770, p. 8) ${ }^{16}$

Magens constate un avantage des néerlandophones - qui ne sont pas forcément des locuteurs natifs - et, plus important encore, il ne doute pas de la nécessité de parler le cariole qui est pourtant considéré comme la langue des esclaves. En même temps, le néerlandais n'est envisagé que comme un relais et non une fin en soi.

À l'inverse, bon nombre de missionnaires des Frères moraves notent dans leurs journaux qu'ils se sont préparés à leur séjour en apprenant le néerlandais pendant le voyage en mer (Stein 1996, p. 32). Le texte d'Oldendorp sème même le doute sur la fonction auxiliaire du néerlandais, en mentionnant un grand nombre d'individus, surtout d'autres Frères moraves, ayant appris le cariole sans maîtriser vraiment le néerlandais ${ }^{17}$. Leur connaissance de l'allemand ou, dans certains cas, du bas-allemand, aura peut-être facilité ce processus. Oldendorp est même d'avis que le néerlandais pourrait nuire à l'acquisition du cariole :

On pourrait croire que celui qui parle hollandais comprendrait par là même de ce chef aussi le créole ou qu'il serait capable de le saisir rapidement parce

16 « Da nu dette Creolske Sprog er deriveret af det Hollandske, saa er det en Fornødenhed, at den der hastig vil lære det første, maa forstaae det sidste; Dette er Aarsagen, at de, som kommer hertil fra Hollandske Stæder, kan snarere lære at omgaaes og forstaae Negerne, end de, som kommer fra andre Steder. »

17 Voir la compilation dans Stein 2010, p. 212-213, note 16. 
qu'il contient tant de mots de cette langue. Pourtant, beaucoup d'exemples dont j'ai entendu parler et d'autres que j'ai vus moi-même prouvent le contraire. Certains ont dit eux-mêmes, et je l'ai remarqué aussi en les observant, que le hollandais les gênait plus qu'il ne leur était utile pour apprendre le créole. Celui qui est encore jeune et qui a une bonne mémoire pourra sans doute apprendre à distinguer entre ces deux langues qui sont très différentes, mais celui qui n'a pas cette faculté y aura de grandes difficultés. Il mélangera très facilement l'une avec l'autre. [...] En croyant parler créole, il parle donc hollandais et personne ne le comprend. [...] Pour apprendre cette langue, il est plus utile de comprendre un peu le hollandais et d'en connaître beaucoup de mots sans savoir le parler habilement. (Oldendorp 2000a [1777], p. 712 [809]) ${ }^{18}$

La description grammaticale qu'Oldendorp donne du cariole témoigne du caractère systématique de son savoir métalinguistique sur le néerlandais. Au début de sa grammaire, Oldendorp discute des différentes possibilités d'écrire le créole. Il écarte le recours à une orthographe purement phonographique car certains esclaves avaient déjà été alphabétisés en néerlandais et certains textes carioles avaient déjà été imprimés dans une graphie néerlandaise. Il opte donc pour cette dernière - choix pratique et non pas idéologique - et donne un aperçu des règles de l'orthographe néerlandaise ${ }^{19}$ appliquée au cariole avec des explications de prononciation pour les lecteurs germanophones (Oldendorp 2000a [1777], p. 684684 [774-775]). Pour ce faire, il devait forcément avoir une certaine maîtrise du néerlandais écrit, ainsi que de l'orthographe et de la prononciation du néerlandais comme du bas-allemand. Né à proximité de Hildesheim, ses connaissances du dialecte local du bas-allemand lui avaient, de fait, facilité l'accès au cariole. Il décrit ainsi son arrivée dans les Antilles danoises :

Dès que mes autres activités me le permirent, je m'occupai de la langue créole. J'ai prononcé le premier discours dans cette langue le dernier juillet devant les Noirs dans l'assemblée de Bethanien à St. Jean, où je demeurais depuis quelques jours. [...] Pour l'étude de cette langue, le bas-allemand ou bas-saxe m'était fort utile. (Oldendorp 2002 [1777], p. 1684 [2aI, 32]) $)^{20}$

18 «Man sollte aber glauben, wer Holländisch könnte, verstände deswegen auch Criolisch oder wäre doch im Stande, es bald zu fassen, weil es von jener Sprache so viele Wörter hat. Allein viele Beispiele, die ich teils gehört, teils selber gesehen habe, beweisen das Gegenteil. Einige haben es selber gesagt, und ich habe es auch bei ihnen bemerket, dass ihnen ihr Holländisch zur Erlernung des Criolischen mehr hinderlich als förderlich war. Wer noch jung ist und ein gut Gedächtnis hat, der kann wohl beide Sprachen, die sehr verschieden sind, auseinanderhalten lernen, wem aber jenes fehlt, dem wird dieses ungemein schwer. Ein solcher mischt leicht das eine in das andere. [...] Er redet also, da er glaubt criolisch zu reden, holländisch, und niemand versteht ihn. [...] Eher hilft es zur Erlernung dieser Sprache, wenn man etwas Holländisch versteht und viele Wörter davon weiß, ohne dass man es fertig reden kann. »

19 L'orthographe n'était certes pas codifiée formellement à l'époque, mais il existait bien un noyau de règles implicites, accompagné d'un niveau élevé de variation.

20 « Sobald ich für anderer dringenden Arbeit dazu kommen konnte, legte ich mich auf die criolische Sprache. Ich hielt die erste Rede in derselben an die Schwarzen in der Versammlung den letzten Julius in Bethanien in St. Jan, wo ich mich damals seit einigen Tagen befand [...]. Zur Erlernung dieser Sprache kam mir das Plattdeutsche oder Niedersächsische sehr zustatten [...]. » 
Il n'est donc pas fait mention du néerlandais, en dépit de l'intérêt d'Oldendorp pour les questions linguistiques. S'il avait appris le néerlandais de façon ciblée et systématique, il l'aurait sans doute mentionné dans ce passage. On notera d'ailleurs que l'itinéraire d'Oldendorp vers les Antilles l'avait d'abord mené à Copenhague où il avait passé cinq semaines, mises à profit pour « se familiariser avec la langue danoise pour pouvoir la lire » (Oldendorp 2002 [1777], p. 1668 [2a1, 13]). Un séjour d'Oldendorp aux Pays-Bas n'est attesté qu'après son voyage aux Îles Vierges (Gilbert 1994, p. 2871).

Outre ces détails biographiques, les connaissances linguistiques d'Oldendorp transparaissent aussi dans ses commentaires sur la structure du cariole. En faisant référence à la prononciation, Oldendorp distingue entre des mots qui sont dérivés du néerlandais et ceux qui seraient, à son avis, d'origine bas-allemande ou bassaxonne :

Comme l'indique la prononciation, des mots bas-allemands ou bas-saxes sont krieg recevoir, kik voir, skriev écrire, bliev rester, bewies prouver, bring apporter, swieg se taire, stri disputer, la dispute, $b i$ chez, $u t$ de, la préposition à signification très large na, hoes maison, Sie le côté, tied le temps et beaucoup d'autres. (Oldendorp 2000a [1777], p. 687 [777]) $)^{21}$

La plupart de ces exemples concernent des mots qui contiennent la voyelle [i] là où le néerlandais standard aurait une diphtongue [ci], indiquée dans l'orthographe actuelle par le digraphe $<\mathrm{ij}>$ comme dans blijven (rester) ou bien $k i$ jken (regarder). Pourtant, on ne peut pas attribuer ces mots au bas-allemand avec une totale certitude car les dialectes flamands et zélandais, variétés décisives dans la formation du cariole, présentent eux aussi de tels traits caractéristiques. (Van Rossem 2000, p. 49). La graphie $<$ hoes $>$ avec le digraphe $<$ oe $>$ pour la voyelle $[\mathrm{u}]$ est précise et cohérente. Le bas-allemand connaît effectivement cette prononciation qui aura donné [hœys] en néerlandais moderne. Pourtant, les dialectes en question pouvaient présenter cette même prononciation en $[\mathrm{u}]$ déjà à l'époque (Van Rossem 2000, p. 9). Ces nuances dialectologiques du néerlandais échappent à Oldendorp. Il faudra attendre la parution du travail très important du Néerlandais Dirk Christiaan Hesseling ${ }^{22}$ en 1905 pour que des analyses plus fines tiennent compte de la variation dialectale dans la formation phonologique et aussi lexicale du cariole (Hesseling 1905, p. 74-99). Entre ces flottements de graphie, de changement phonologique et de variation linguistique historique et diatopique, il est

21 «Plattdeutsch oder niedersächsisch ist, wie die Aussprache anzeigt, krieg kriegen, bekommen, kik sehen, skriev schreiben, bliev bleiben, bewies beweisen, bring bringen, swieg schweigen, stri streiten, der Streit, bi bei, ut aus, die nach der Bedeutung sehr ausgedehnte Präposition na, hoes Haus, Sie die Seite, tied die Zeit und viel anderes. »

22 Voir Noordegraaf $(2013,2014)$ pour une vue d'ensemble du travail de Hesseling dans le domaine de la néerlandistique extra-européenne et dans la créolistique néerlandaise au début $\mathrm{du} \mathrm{XIX}^{\mathrm{ES}}$ 
extrêmement difficile d'identifier avec exactitude les compétences d'Oldendorp. Il s'exprime d'ailleurs lui-même avec une grande prudence sur l'étymologie des mots du cariole, sans pour autant tenir compte des variations dialectales possibles :

Le hollandais et le bas-allemand ont tant de mots en commun, et aussi avec l'anglais et le danois, que très souvent on ne sait pas de laquelle de ces langues provient un mot créole et comment il faudrait alors l'écrire. (Oldendorp 2000a [1777], p. 683 [773] $)^{23}$

Oldendorp accorde cependant une place importante à l'allemand, soit dans la forme bas-allemande, soit dans la forme standardisée :

Dans les cantiques, on a parfois fait un génitif allemand au singulier au profit du mètre ou de la rime, comme God Vaders kind; joe Geestes hand de la main de Ton Esprit; Lam Gods ou Lam Godes. (Oldendorp 2000a [1777], p. 690 [781]) ${ }^{24}$

À l'époque, une telle forme était aussi possible en néerlandais dans un texte formel religieux. L'auteur de cette tournure peut avoir recouru à une structure allemande ou bien néerlandaise. Il ne faut pas oublier que toutes les traductions des textes liturgiques dont Oldendorp disposait avaient été produites par des missionnaires allemands. Oldendorp était conscient de ces interférences dans le processus de traduction, ainsi que des particularités du style écrit et formel des textes religieux. En la matière, Oldendorp souligne d'ailleurs l'importance d'une bonne connaissance du néerlandais - alors même qu'il la jugeait gênante pour l'acquisition du cariole :

Dans la traduction de certains passages de l'Écriture sainte, il s'avère aussi parfois que des mots manquent. Ceux-ci doivent alors être introduits si une périphrase commode ne peut pas prendre leur place. Et on les prend le plus naturellement dans la langue hollandaise, quelques fois aussi dans l'allemande, et on leur donne une ressemblance au créole, s'ils en manquent. Mais pour ce faire il faut de la prudence et une bonne connaissance de la langue hollandaise afin de ne pas fabriquer, à partir de leur ressemblance avec d'autres, des mots qui de ce fait ne signifieraient pas toujours en hollandais ce qu'ils devraient, mais souvent plutôt quelque chose de très différent. (Oldendorp 2000a [1777], p. $715[811])^{25}$

23 « Das Holländische und Plattdeutsche hat so viele Wörter miteinander, auch nicht wenige mit dem Englischen und Dänischen gemein, dass man bei einem criolischen Wort oft nicht weiß, von welcher dieser Sprachen es herkommt und wie es also eigentlich geschrieben werden müsse. »

24 « In Kirchengesängen ist bisweilen um des Silbenmaßes oder Reims willen ein deutscher Genitivus in der einzeln Zahl gemacht worden, als God Vaders kind ; joe Geestes hand deines Geistes Hand ; Lam Gods oder Lam Godes. »

25 « Bei der Übersetzung mancher Stellen der heiligen Schrift und geistlichen Lieder zeigt es sich noch bisweilen, dass Wörter dazu fehlen. Solche müssen denn eingeführt werden, wenn nicht eine bequeme Umschreibung ihre Stelle versehen kann. Und man nimmt sie am natürlichsten aus der holländischen, auch bisweilen aus der deutschen Sprache und richtet sie nach der Ähnlichkeit der criolischen ein, wenn ihnen daran etwas mangelt. Dazu ist aber Behutsamkeit und eine gute Wissenschaft der holländischen Sprache nötig, damit man nicht nach der Ähnlichkeit anderer Wörter welche mache, die deswegen im Holländischen nicht immer heißen, was sie heißen sollen, sondern oft viel was anders bedeuten. » 
Si le néerlandais est donc déconseillé aux débutants, le traducteur-missionnaire doit le maîtriser pour pouvoir créer des néologismes sans tomber dans le piège des faux-amis ou des connotations inopportunes.

Quant à Magens, son texte offre beaucoup moins de pistes pour déterminer son degré de connaissance du néerlandais. Quelques indications éparses laissent néanmoins supposer qu'il avait, comme Oldendorp, une notion des relations entre l'orthographe et la prononciation néerlandaises. Les explications sur la prononciation du cariole sont très courtes, mais elles contiennent une information essentielle :

Chaque lettre se prononce dans la langue créole avec le son complet, comme en danois. Le $\mathrm{V}$ uniquement se prononce comme $\mathrm{F}$ pour la raison mentionnée plus haut, à savoir qu'elle est dérivée du hollandais, et par conséquent, on utilise W à la place de V, comme en haut-allemand. (Magens 1770, p. 9) ${ }^{26}$

La certitude avec laquelle Magens se prononce ici sur l'origine historique de la prononciation cariole est pour le moins surprenante. La désonorisation de $[\mathrm{v}]$ vers [f] en néerlandais est en effet un développement assez récent observé plus fréquemment depuis le $\mathrm{XX}^{\mathrm{e}} \mathrm{s}$. et qui s'est répandu, en partant justement des dialectes de la Hollande, notamment dans le nord du territoire néerlandophone européen (Van de Velde / Gerritsen / Van Hout 1995). La prononciation sourde de la fricative labiodentale est aujourd'hui une particularité du néerlandais moderne aux Pays-Bas tandis que les dialectes flamands et la prononciation courante du néerlandais standard en Belgique ont conservé une fricative sonore. Il est difficile de déterminer si les dialectes de la Zélande connaissaient ce trait particulier au XVII ${ }^{\mathrm{e}}$ ou au XVIII ${ }^{\mathrm{e}} \mathrm{s}$. Les observations historiques de ce phénomène sont assez ambigües (Kooij/Van Oostendorp 2003, p. 62-63). Comme Oldendorp, Magens tient à signaler à ses lecteurs une différence importante entre le danois et le néerlandais qui leur sera utile pour se familiariser avec la prononciation du cariole. Il est bien possible que la désonorisation décrite par Magens soit un développement autonome du cariole survenu dans le processus de créolisation. Dans ce cas-là, cependant, l'explication de Magens serait évidemment erronée. Nous ignorons en tout cas si Magens était au courant de ces nuances dialectales du néerlandais ou bien si l'image qu'il se fait de la langue néerlandaise - ou celle que s'en font ses informateurs néerlandophones locaux - a pu être influencée par la prononciation hollandaise. Ce que Magens entend par « hollandais » est au demeurant imprécis, puisque ce terme est utilisé couramment pour désigner le néerlandais en général aussi bien que les dialectes de la Hollande.

Pontoppidan, à son tour, ajoute une collection de proverbes à son article. C'est cette partie du texte qui trahit une petite incertitude dans le détail d'une traduction :

26 «Hver Bogstav udtales i de Creolske Sprog med sin fulde Lyd, som i det Danske. Alleneste $\mathrm{V}$ udtales som $\mathrm{F}$ af føranførte Aarsag, at det er deriveret af det Hollandske, og følgelig bruges W i steden for V, som i det Høytydske. » 
Twee slem mens no kan kok Boontje na een Pot.

Deux personnes rusées/méchantes ne peuvent pas cuir des haricots/petits pois dans un seul pot.

[Origin. : To onde Mennesker kan ikke koge Ærter i en Gryde.]

(Pontoppidan 2014 [1887], p. 96 [303])

Pontoppidan ne reconnaît pas l'origine probable du mot slem, dérivé du néerlandais slim (rusé), et le confond avec le danois slem (méchant) ${ }^{27}$. En même temps, la traduction danoise comprend une petite imprécision car Pontoppidan traduit Boontje (haricots) par Arter (petits pois). Même mineures, ces confusions suffisent à montrer que Pontoppidan ne saisit pas toutes les nuances de l'étymologie cariole ni de ses racines lexicales néerlandaises. Il reste à déterminer si la signification de ce proverbe n'est pas plus claire avec le terme méchant qu'avec celui de rusé.

\section{LE CRÉOLE 《 ESSENTIEL 》 :}

\section{ENTRE UNIVERSALISME ET SUPÉRIORITÉ DU NÉERLANDAIS}

Les remarques métalinguistiques d'Oldendorp nous renseignent non seulement sur ses compétences linguistiques et la situation sociolinguistique des Antilles danoises, mais aussi sur sa façon de concevoir le créole et le langage humain en général. Oldendorp s'exprime sur les qualités du cariole aussi bien que sur ses locuteurs. Ses observations ethnographiques sur le physique et les mœurs des esclaves s'inscrivent dans la logique contemporaine de l'exotisme colonial. Cependant, cette attitude coloniale ne se traduit pas encore par un déterminisme absolu mais s'ancre plutôt dans un universalisme typique de la mission chrétienne (Krämer 2012). Le rapport d'Oldendorp abonde en passages qui soulignent la prétendue nécessité mais aussi la possibilité d'éduquer les esclaves. Ainsi, le créoliste allemand ne cesse-t-il d'insister sur la capacité impressionnante des « Noirs » à apprendre des langues.

Si le cariole est, pour Oldendorp comme pour beaucoup d'autres Européens de l'époque, une langue réputée simple et facile à apprendre, son acquisition dépend plus des capacités ou de la volonté de l'individu que de prédispositions collectives, par exemple raciales. Ce ne sera qu'à la fin du siècle suivant avec les travaux de Pontoppidan et de nombreux autres créolistes de l'époque, que le critère de la différence raciale jouera un rôle prépondérant (Krämer 2013, 2014). Si le médecin danois reconnaît lui aussi la capacité des «Noirs» à apprendre des langues, il n'hésite pas à relativiser immédiatement cette assertion :

Toute personne ayant vécu parmi des nègres saura avec quelle aisance ils apprennent à utiliser une langue étrangère. Mais cette aisance d'acquisition ne dépasse pas un certain point. [...] La grammaire leur pose des difficultés

27 Voir aussi la traduction anglaise comme « smart » dans Van Rossem / Van der Voort (1996, p. 212). 
insurmontables, même dans la langue anglaise qui est si simple et informe. (Pontoppidan 2014 [1887], p. 91 [289]) ${ }^{28}$

Contrairement à Oldendorp, Pontoppidan n'établit pas de distinctions entre les individus. Tous les « Noirs » seraient limités dans leur acquisition linguistique. Cette limitation se traduirait aussi dans les proverbes créoles, dont la matière serait tirée uniquement de l'expérience quotidienne immédiate et qui, « utilisés de façon répétitive, épargne(raie)nt à l'intelligence faible et inexpérimentée l'effort de former de nouvelles pensées et expressions. (Pontoppidan 2014 [1887], p. 92 [299]). Les proverbes ne seraient pas seulement le signe d'une intelligence limitée des « Noirs » mais aussi de leur façon de penser, de leur « esprit ». ${ }^{29}$

Magens et Oldendorp donnent également des proverbes dans leurs travaux ${ }^{30}$. Les deux créolistes du xviii ${ }^{\text {e }}$ s., par contre, ne vont pas aussi loin dans l'interprétation des proverbes comme indice d'un esprit collectif. Tout comme les autres textes créoles tels que les dialogues exemplaires ou les textes liturgiques qu'ils publient dans leurs travaux, les proverbes sont avant tout censés illustrer l'usage quotidien de la langue et ses structures, étant donné que les grammaires ne contiennent que des passages très courts et non pas des énoncés ou des textes plus développés.

En dépit de différences très nettes dans leurs orientations discursives et conceptuelles, les trois travaux ont en commun de vouloir démontrer une « essence » du cariole, de montrer ce qui est typique. Pour Oldendorp et Magens, cette essence est plutôt celle d'un usage linguistique, pour Pontoppidan, par contre, il s'agit d'une essence raciale. Dans les deux cas, le néerlandais sert d'objet de référence ou de comparaison:

Comme on ne peut pas très bien apprendre, de ce qui précède, les vrais proverbes créoles qui s'utilisent dans la vie quotidienne, parce que les proverbes théologiques et les mots courants qu'ils contiennent sont formés pour la plupart sur le hollandais, j'ai collectionné les proverbes suivants [...]. (Magens 1770, p. 51$)^{31}$

Les Noirs comprennent de tels verbes passifs bien qu'ils ne les utilisent pas souvent. Mais il ne faut pas en faire trop parce qu'on parlera alors de manière

28 « Enhver, der har levet imellem Negere, ved ogsaa, med hvilken Lethed de lære at benytte et fremmed Sprog. Men Letheden i Tilegnelsen gaar kun til et vist Punkt. [...] Grammatiken gør dem uovervindelige Vanskeligheder, endogsaa i det saa simple og formløse engelske Sprog. »"

29 Pour l'importance des notions d' « esprit » et de « génie » dans la créolistique du XIX ${ }^{e}$ S., voir Krämer (2014, p. 184-185), voir aussi Meschonnic (1997) pour une vue d'ensemble très complète de cette conception centrale dans l'histoire de la philosophie linguistique.

30 Voir von Sicard (2014) pour une analyse détaillée des proverbes chez les trois auteurs et leur position dans la littérature orale créole.

31 « Da man ikke ret vel af forestaaende Samtale kand lære de egentlige Creolske Talemaader, som bruges i den daglige Omgang, af Aarsage, at de Theologiske Talemaade og de dertil brugelige Ord ere mestendeel efter det Hollandske, saa har jeg sammenskrevet følgende Samtaler [...]. » 
trop a-créole (Oldendorp 2000 [1777], p. 700 [794]). ${ }^{32}$

À côté de ces restes de la langue vivante et parlée, il faut certainement se tourner vers la littérature haut-créole, mais ceci doit être considéré, à côté de la langue nègre propre et originelle, comme quelque chose d'appris et d'artificiel que les nègres comprennent à peine et qui très souvent ne devient que du mauvais hollandais. [...] C'est pourquoi je prends en compte, dans ce qui suit, notamment le vrai créole tel qu'il se parle ou plutôt se parlait dans les échanges ordinaires [...]. (Pontoppidan 2014 [1887], p. 91 [297-298]) ${ }^{33}$

Le créole considéré comme «typique » est toujours celui qui est le plus distant du néerlandais. Le néerlandais devient, pour ainsi dire, la négation du cariole, ou vice versa. L'opposition imaginaire entre le néerlandais et le créole est absolue bien que les deux structures restent en réalité ouvertes l'une à l'autre, notamment dans le domaine des emprunts, fait que les trois créolistes admettent plus ou moins explicitement ${ }^{34}$. Cependant, cette opposition ne s'explique pas par une loyauté accrue envers le néerlandais, étant donné qu'aucun des trois auteurs n'est d'origine néerlandaise ni locuteur natif de la langue. Le statut du néerlandais est tout d'abord d'être la langue de base du cariole et, en même temps, il représente tout ce qui est européen. Les auteurs ne privilégient donc pas le néerlandais pour son statut de langue "parfaite» ou "supérieure » mais tout simplement en raison de sa fonction historique et structurelle pour le cariole. C'est notamment en tant que source d'emprunts lexicaux et dans certains cas aussi grammaticaux ${ }^{35}$, par exemple dans le domaine liturgique et religieux, que le néerlandais trouve sa place dans la pratique linguistique des créolistes. Dans cette fonction, ils lui accordent un avantage sur le cariole. Dans les propos d'Oldendorp, du moins, il est évident que cet avantage n'est pas intrinsèque mais résulte du développement historique et littéraire du néerlandais (voir aussi Stein 2010, p. 225-226) : une telle histoire manquerait au cariole, obligeant à des emprunts pour exprimer des conceptions abstraites, religieuses et philosophiques.

Comme ces trois créolistes ne sont pas d'origine néerlandaise, ils n'ont pas besoin de défendre leur propre langue au même point que d'autres créolistes. Ils

32 «Die Schwarzen verstehen solche passive Verba, ob sie gleich selber sie wenig gebrauchen. Man muss aber deren nicht zu viele machen, sonst redet man zu uncriolisch. »

33 « Ved Siden af disse Rester af det levende, talte Sprog har man saa ganske vist den højkreolske Literatur at gaa til, men dette maa ved Siden af det egentlige og oprindelige Negersprog betragtes som noget tillært og tillavet, der af Negerne knapt forstaas og som paa sine Steder mere bliver til et daarligt Hollandsk. [...] Jeg skal ederfor ogsaa i det følgende tage særligt Hensyn til det egentlige kreolske, saaledes som det tales eller maaske rettere taltes i almindelig Omgang [...]. »

34 À propos de la variation interne du cariole, voir Stein (2013, p. 56-57 et 74).

35 On notera par exemple des discussions autour de la voix passive chez Oldendorp et aussi chez Magens, construction grammaticale atypique dans un registre informel mais souvent jugée nécessaire par les missionnaires dans les traductions des textes religieux (voir aussi l'analyse dans Van Rossem / Van der Voort 1996, p. 38-40 et Stein 2013, p. 70-71). 
arrivent à créer une certaine distance entre leurs langues nationales ou natives et leur objet d'étude. C'est d'autant plus important pour le travail de Pontoppidan. En effet, celui-ci s'inscrit bien dans le discours hiérarchique racialiste de l'époque mais décrit une langue créole moribonde qui n'est donc pas considérée comme menace immédiate pour la prétendue supériorité linguistique et morale des Européens, qu'ils soient néerlandophones ou danophones. On comprend dès lors pourquoi, dans le titre de son article danois, Pontoppidan nomme le cariole la « langue créole dano-antillaise » (dansk-vestindisk Kreolsprog) et que dans le texte, il affirme que cette langue "porte toujours la désignation de dano-créole » (Pontoppidan 2014 [1887], p. 89 [295]) ${ }^{36}$. Il était pourtant bien conscient de la base néerlandaise de cette langue. Jusqu'à présent, aucune langue créole à base danoise n'a été documentée, et les pidgins scandinaves attestés se sont formés presque uniquement dans les régions arctiques et nordiques (Bakker 2003). Une telle stratégie d'appropriation terminologique serait impossible si Pontoppidan avait eu besoin d'une séparation épistémologique claire et nette entre la langue des colonisés et la sienne. Il est du reste assez improbable que le terme « dano-créole » ait été aussi courant que Pontoppidan veut le faire croire (Stein 2010, p. 113), vu le statut marginal du danois dans la colonie.

\section{De la mission À La Dialectologie :}

LE CARIOLE ET LA NÉERLANDISTIQUE EXTRA-EUROPÉENNE

Bien que le néerlandais soit la langue de base principale du cariole, qu'il lui fournisse donc la majorité de son vocabulaire et qu'il reste ainsi l'élément le plus visible dans l'histoire complexe de cette langue créole, il joue un rôle assez limité dans son environnement social et scientifique. Quoique les métaphores généalogiques restent douteuses pour décrire la genèse et le développement d'une langue, on pourrait dire que le néerlandais, en tant que «mère » du cariole, a abandonné son « enfant» dès la naissance.

Cela vaut sur le plan sociolinguistique de la même façon que sur le plan des recherches scientifiques. Le néerlandais ne sert pas de ressource auxiliaire pour l'acquisition du créole ni de ressource communicative interethnique dans l'île. La langue de base est limitée au seul rôle de fournisseur lexical si des emprunts ou néologismes s'avèrent nécessaires. Le néerlandais ne sert pas non plus, jusqu'au début du $\mathrm{XX}^{\mathrm{e}} \mathrm{s}$., de médium de description scientifique du cariole, contrairement par exemple au papiamentu dont on trouve bien des descriptions et des documentations

36 Dans le titre de son article allemand paru quelques années avant (Pontoppidan 1881), Pontoppidan se limite à la désignation plus neutre et plus précise « la langue créole des îles dano-antillaises » («die Kreolensprache der dänisch-westindischen Inseln »). 
néerlandaises publiées dès le $\mathrm{XIX}^{\mathrm{e}} \mathrm{s} \cdot{ }^{37}$. Même si la dialectologie connut un essor énorme au $\mathrm{XIX}^{\mathrm{e}} \mathrm{s}$., dans la sphère néerlandophone comme ailleurs, cet intérêt n'engendra pas encore de production scientifique sur les variétés caribéennes du néerlandais. Ce n'est qu'en 1905 que parut le travail de Dirk Christiaan Hesseling et donc la première grande description du cariole en néerlandais par un Néerlandais. Pourquoi la philologie néerlandaise de cette époque ne s'intéresset-elle pas au cariole ? Une raison très simple pourrait être le manque d'échanges entre les Îles Vierges et les Pays-Bas vers la fin du XIX ${ }^{e}$ s., période décisive pour le développement de la créolistique comme domaine d'études académiques (Krämer 2014, p. 190, 207). Le colonialisme néerlandais avait déjà dépassé son apogée et une langue créole presque moribonde, parlée par un très petit nombre de locuteurs dans une colonie danoise, n'était sans doute connue que de quelques philologues ou linguistes néerlandais.

En effet, l'intérêt pour les langues créoles était dû très souvent aux passions ou aux expériences personnelles. En 1840, le philologue et historien-archiviste Laurens Philippe Charles van den Bergh publia une petite note d'à peine deux pages pour signaler cette langue à ses collègues, mais l'appel ne fut pas suivi de travaux plus conséquents. Van den Bergh lui-même se limite à la mention de quelques travaux publiés en créole et de deux petites indications grammaticales ainsi qu'à une reproduction du Notre Père selon le Mithridates d'Adelung. En dépit de cette très faible production philologique néerlandaise sur le cariole au $\mathrm{XIX}^{\mathrm{e}} \mathrm{s}$., cette petite note est la première attestation du terme Negerhollands, désignation qui s'est maintenue jusqu'à aujourd'hui.

On notera ainsi une différence très importante entre l'histoire du cariole et celle de la description de la plupart des autres langues créoles. Les travaux décrivant les langues créoles à base française sont presque exclusivement publiés en français par des créolistes originaires de France ou de la sphère coloniale française. À quelques exceptions près - dont celle très importante de l'œuvre de Hugo Schuchardt - le même constat s'applique aussi à la créolistique portugaise (Sousa 2007).

Le néerlandais reste donc un élément à la fois constitutif et marginal dans l'histoire sociolinguistique et philologique des Îles Vierges. L'existence-même du cariole et les circonstances dans lesquelles il était parlé et décrit sont singulières eu égard tant à la philologie coloniale que, plus largement, à l'histoire des sciences du langage.

37 Voir par exemple Fredriks (1859), rééd. par Johannes Kramer (2009), Jesurun (1897, 1898), ce dernier rééd. dans Noordegraaf (2015). 


\section{RÉFÉRENCES}

Bakker, Peter, 2003. «Scandinavians and their pidgins and creoles », Acta Linguistica Hafniensia - International Journal of Linguistics 35/1, 95-114.

Frederiks, Bernardus Th. J., 1859. Woordenlijst der in de landstaal van Curaçao meest gebruikelijke woorden, rééd. 2009 par Johannes Kramer, Hambourg, Buske.

Gilbert, Glenn G., 1994. « Oldendorp, Christian Georg Andreas (1721-1787) », R.E. Asher et J.M.Y. Simpson (ed.), The Encyclopedia of Language and Linguistics, Vol. 5, Oxford, New York etc., Pergamon Press, 2871-2872.

Hesseling, Dirk Christiaan, 1905. Het Negerhollands der Deense Antillen. Bijdrage tot de geschiedenis der Nederlandse taal, Leyde, Sijthoff (rééd. DBNL 2006).

Holm, John, 2000. An introduction to pidgins and creoles, Cambridge, Cambridge University Press.

Jesurun, Abraham Daniel, 1897. « Eenige beschouwingen over de volkstaal van Curaçao », Eerste jaarlijksch verslag van het geschied-, taal-, land- en volkenkundig genootschap, gevestigd te Willemstad, Curaçao, Amsterdam, J.H. de Bussy, 95-110.

- 1898. "Het Papiëmentsch », Tweede jaarlijksch verslag van het geschied-, taal-, landen volkenkundig genootschap, gevestigd te Willemstad, Curaçao, Amsterdam, J.H. de Bussy, 75-102. (Rééd. dans Noordegraaf 2015, p. 8-27).

Kooij, Jan et van Oostendorp, Marc, 2003. Fonologie: uitnodiging tot de klankleer van het Nederlands, Amsterdam, Amsterdam University Press.

Krämer, Philipp, 2012. « Das Universalistische Sprachbild bei Oldendorp und Caulier: Frühe Kreolistik deutscher und französischer Missionare », Busch, Anna, Hengelhaupt, Nana et Winter, Alix (eds), Französisch-Deutsche Kulturräume um 1800: Bildungsnetzwerke - Vermittlerpersönlichkeiten - Wissenstransfer, Berlin, Berliner Wissenschafts-Verlag, 283-295.

- 2013. «Linguistique coloniale au XIX ${ }^{\mathrm{e}}$ siècle: Le discours racialiste dans la recherche française sur les langues créoles », French Colonial History 14, 55-70.

- 2014. Die französische Kreolistik im 19. Jahrhundert. Rassismus und Determinismus in der kolonialen Philologie, Hambourg, Buske (Kreolische Bibliothek, Vol. 25).

Lampe, Armando, 2001. Mission or submission? Moravian and catholic missionaries in the Dutch Caribbean during the 19th century, Gœttingue, Vandenhoeck \& Ruprecht.

Magens, Jochum Melchior, 1770. Grammatica over det Creolske Sprog, som bruges paa de trende Danske Eilande, St. Croix, St. Thomas og St. Jans i America. Sammenskrevet og opsat af en paa St. Thomas Indföd Mand, Copenhague, Det Kongelige Waeysenhusets Bogtrykkerie.

Markey, Thomas L., 1982. « Afrikaans: creole or non-creole? », Zeitschrift für Dialektologie und Linguistik 49, 169-207.

Meschonnic, Henri, 1997. De la langue française. Essai sur une clarté obscure, Paris (rééd. 2001), Hachette.

Noordegraaf, Jan, 2013. « Hesseling honderd jaar later. Over postkoloniale linguïstiek in Nederland », Janssen, Theo et Noordegraaf, Jan (ed.), Honderd jaar taalwetenschap. Artikelen aangeboden aan Saskia Daalder bij haar afscheid van de Vrije Universiteit, Münster, Nodus, 127-138.

- 2014. " Verre verwanten. D.C. Hesseling (1859-1941) over taal en taalverandering », Freek Van de Velde et al. (ed.), Patroon en argument. Een dubbelfeestbundel bij het emeritaat van William van Belle en Joop van der Horst, Louvain: Presses Universitaires, 523-535.

- 2015. "Lexicografie op Curaçao. Abraham Jesurun en zijn woordenlijst van het Papiaments (1898)", Trefwoord, tijdschrift voor lexicografie, 2015. En ligne : https://www.fryske-akademy.nl/nl/fryske-akademy/utjeften/trefwoord/jaargang-2015.

Oldendorp, Christian Georg Andreas, 2000a [1777]. Historie der caribischen Inseln Sanct Thomas, Sanct Crux und Sanct Jan, insbesondere der dasigen Neger und der Mission der Evangelischen Bruder unter denselben, 1ère partie. Rééd. par Gudrun Meier, Stephan Palmié, Peter Stein, Horst Ulbricht, Berlin, Verlag für Wissenschaft und Bildung. 
— 2002 [1777]. Historie der caribischen Inseln Sanct Thomas, Sanct Crux und Sanct Jan, insbesondere der dasigen Neger und der Mission der Evangelischen Bruder unter denselben, $2^{\mathrm{e}}$ partie. Rééd. par Gudrun Meier, Stephan Palmié, Peter Stein, Horst Ulbricht. 3 volumes. Berlin, Verlag für Wissenschaft und Bildung.

— 2010 [1787]. « Lebenslauf des am 9. Merz 1787 in Ebersdorf heimgegangenen Bruders Christian Georg Andreas Oldendorp », Meier, Gudrun, Stein, Peter, Palmié, Stephan et Ulbricht, Horst (éd.), Christian Georg Andreas Oldendorp. Historie der caribischen Inseln Sanct Thomas, Sanct Crux und Sanct Jan. Kommentarband, Herrnhut, Herrnhuter Verlag, 1-8.

Pontoppidan, Erik, 1881. "Einige Notizen über die Kreolensprache der dänischwestindischen Inseln », Zeitschrift für Ethnologie 13, 130-138. Rééd. dans Krämer, Philipp (éd., 2014), Ausgewählte Arbeiten der Kreolistik des 19. Jahrhunderts. Emilio Teza, Thomas Russell, Erik Pontoppidan, Adolpho Coelho, Hambourg, Buske, 79-87.

- 1887. « Det dansk-vestindiske Kreolsprog », Tilskueren 4, 195-303. Rééd. dans Krämer, Philipp (éd., 2014), Ausgewählte Arbeiten der Kreolistik des 19. Jahrhunderts. Emilio Teza, Thomas Russell, Erik Pontoppidan, Adolpho Coelho, Hambourg, Buske, 89-97.

Sabino, Robin, 2012. Language Contact in the Danish West Indies: Giving Jack His Jacket, Leyde, Brill.

Stein, Peter, 1996. Einleitung / C.G.A. Oldendorps Criolisches Wörterbuch, Oldendorp, Christian Georg Andreas, 1996 [1767/68], Criolisches Wörterbuch. Erster zu vermehrender und wo nöthig zu verbessernder Versuch, éd. et commenté par Peter Stein, Tübingen, Niemeyer, 1-38.

- 1998. «Oldendorp, Christian Georg Andreas », Brekle, Herbert E. et al. (eds), Biobibliographisches Handbuch zur Sprachwissenschaft des 18. Jahrhunderts. Die Grammatiker, Lexikographen und Sprachtheoretiker des deutschsprachigen Raums mit Beschreibungen ihrer Werke, Vol. 6, Tübingen, Niemeyer, 369-373.

- 2002. « C. G. A. Oldendorp, chroniqueur de la vie coloniale au milieu du XVIII siècle, et le sort de son Histoire de la mission des frères évangéliques aux îles caraïbes de SaintThomas, Saint-Jean et Sainte-Croix », Pelletier, Monique (éd.), Les îles, du mythe à la réalité $\left[123^{e}\right.$ Congrès national des sociétés historiques et scientifiques, Fort-de-France, 4-11 avril 1998], Paris, Éds. du CTHS, 181-188.

- 2010. "Oldendorp und das Kreolische », Meier, Gudrun, Stein, Peter, Palmié, Stephan et Ulbricht, Horst (eds), Christian Georg Andreas Oldendorp. Historie der caribischen Inseln Sanct Thomas, Sanct Crux und Sanct Jan. Kommentarband, Herrnhut, Herrnhuter Verlag, 207-247.

- 2013. « Histoire d'une langue créole, le Negerhollands, 1736-1985, suivie d'une étude du début de la grammaticographie créole: C.G.A. Oldendorp », Pourchez, Laurence (ed.), Créolité, créolisation: regards croisés, Saint-Denis de la Réunion, Éditions des archives contemporaines, 53-78.

- 2014. « Dr. med. Erik Pontoppidan und das Negerhollands », Krämer, Philipp (ed.), Ausgewählte Arbeiten der Kreolistik des 19. Jahrhunderts. Emilio Teza, Thomas Russell, Erik Pontoppidan, Adolpho Coelho, Hambourg, Buske, 109-121.

Sousa, Silvio Moreira de, 2007. A Teoria Crioula de Adolfo Coelho segundo a Correspondência com Hugo Schuchardt e Leite de Vasconcelos, Vienne, Université de Vienne (mémoire de maîtrise).

Roberge, Paul T., 2002. "Afrikaans: considering origins », Rajend, Mesthrie (ed.), Language in South Africa, Cambridge, Cambridge University Press, 79-103.

Rupp-Eisenreich, Britta, 1985. « Les Frères Moraves, ethnologues de la condition esclave ? (Îles Vierges, Petites Antilles, 1731-1768)», Blanckaert, Claude (éd.), Naissance de l'ethnologie? Anthropologie et missions en Amérique, XVI ${ }^{e}-X V I I I^{e}$ siècles, Paris, Cerf, 125-173.

Van den Bergh, Laurens Philippe Charles, 1840. "Iets over het Neger-Hollandsch », Taalkundig Magazijn of gemengde bijdragen tot de kennis der Nederduitsche taal [éd. par A. de Jager], Vol. 3, 4 partie, 500-501. 
Van de Velde, Hans, Gerritsen, Marinel et van Hout, Roeland, 1995. « De verstemlozing van de fricatieven in het Standaard-Nederlands. Een onderzoek naar taalverandering in de periode 1935-1993 », De Nieuwe Taalgids 88, 422-445.

Van Rossem, Cefas et van der Voort, Hein, 1996. Die Creol taal. 250 years of Negerhollands texts, Amsterdam, Amsterdam University Press.

Van Rossem, Cefas, 2000. "Het Negerhollands, Negerzeeuws, Negervlaams ", Berns, Johannes B. et van Marle, Jaap (eds), Overzees Nederlands, Lezingen gehouden op het symposion van de afdeling Dialectologie van het P.J. Meertens-Instituut op vrijdag 26 november 1993, Amsterdam, Meertens Instituut, 40-62.

— 2013. «Maternity visit on St. Thomas. Demographic information on the earliest stages of Negerhollands ", Revue Belge de Philologie et d'Histoire 91, 713-734.

von Sicard, Magdalena, 2014. «Die kreolische Oralliteratur: Eine Kultur des Widerstandes? », Krämer, Philipp (ed.), Ausgewählte Arbeiten der Kreolistik des 19. Jahrhunderts. Emilio Teza, Thomas Russell, Erik Pontoppidan, Adolpho Coelho, Hambourg, Buske, 123-142. 